\title{
Biomarkers and Obesity
}

\section{Emanuele Lo Menzo*}

Department of Surgery, Section Bariatric and Laparoscopic Surgery, University of Maryland School of Medicine, 22 S. Greene St, Room N4E27 Baltimore, Maryland, USA

Obesity is now recognized as a worldwide epidemic, and its incidence is increasing exponentially. According to the World Health Organization's projections of 2005 approximately 1.7 billion people are considered overweight, and at least 400 million adults are obese. In the US alone, nearly $33 \%$ of the population ( 97 million) is obese and approximately 10 million people are morbidly obese (or class III obesity BMI $\geq 40$ ). Unfortunately the problem seems to have deep roots. In fact, according to the National Center for Health Statistics of the CDC, approximately $17 \%$ (or 12.5 million) of children and adolescents aged 2-19 years are obese in the US.

Obesity remains one of the most prominent risk factors for cardiovascular diseases, as well as, for specific cancers. Do we have any biomarkers that help us determine the cardiovascular and cancer risk factors in the obese population?

Cardiovascular diseases derive from an often silent progression of the atherosclerotic process, hence the importance of an early diagnosis. Since morbid obesity is characterized by a chronic inflammatory state, an array of inflammatory biomarkers result chronically dysregulated secondary to the altered endocrine function of the adipocytes. Typically pro-inflammatory cytokines and chemokines, such as TNF- $\alpha$, ICAM1, IL-6, VCAM-1, are up regulated in obesity. Whereas other antiinflammatory mediators, such as adiponectin are typically down regulated.

The presence of up regulated cytokines and the increased availability of free fatty acids (FFA) contribute to endothelial dysfunction and vasoconstriction, leading, ultimately, to foam cell formation and atherosclerosis. In addition, obesity also determines increased insulin resistance by increasing FFA availability and reduction of adiponectin, worsening the atherosclerotic process. Finally, hypertension and dyslipidemia complete the picture of the metabolic syndrome.

From this brief overview results obvious how several potential biomarkers could play a role in early diagnosis of cardiovascular disease. Unfortunately studies on these biomarkers are still producing non-convincing results. For instance, adiponectin has been inversely correlated to the risk of cardiovascular diseases in men, but not in women. Leptin, a direct modulator of pancreatic $\beta$-cell function, has been shown to be an independent predictor of diabetes type 2 development in men, but not in women. In other studies, Leptin has been shown to be a predictor of cardiovascular diseases, although opposite results have been reported.
The association between obesity and cancer has been also extensively studied, and oncologic biomarkers have been linked to obesity.

This association is particularly strong for the development of cancers of the esophagus, breast (postmenopausal), endometrium, colon and rectum, kidney, pancreas, and gallbladder. Multiple potential mechanisms have been postulated to explain this association. The decrease production of adiponectin, an anti-inflammatory and anticarcinogen, both systemically and locally, has been associated with the development of post-menopausal breast cancer. In addition, as shown by Sauter et al. [2] the local and systemic adiponectin levels increase inversely proportionally to the weight loss after gastric bypass surgery, offering a potential risk reduction. In the same study, another locally expressed breast specific biomarker, nPSA, resulted inversely related to weight loss, and as such, could represent a protective factor against the development of postmenopausal breast cancer. On the contrary, the increased levels of leptin will promote cell proliferation. Obviously, the excess of peripheral fat produced estrogen also represent a potential explanation for the increased incidence of breast and endometrial cancers.

Obese people often will express increased levels of insulin, secondary to insulin resistance, and insulin-like growth factor-1 (IGF1), which may promote cell proliferation and carcinogenesis.

Although many biomarkers have been identified and linked to different disease processes, the complexity and interaction of different cascades is such that further research is necessary before any of these markers could reach an accurate diagnostic value.

This is why open access publications with their characteristics of immediate free worldwide access to electronic format, are an ideal platform that can provide a real-time snapshot of the available research and help scientists progress in this field.

\section{References}

1. Roos CJ, Quax PH, Jukema JW (2012) Cardiovascular metabolic syndrome mediators involved in the pathophysiology from obesity to coronary heart disease. Biomark Med 6: 35-52.

2. Sauter ER, Mitchell JE, Kliethermes B, Crosby RD (2012) Breast cancer biomarkers predict weight loss after gastric bypass surgery. BMC Res Notes 5: 82 .

*Corresponding author: Emanuele Lo Menzo, Department of Surgery, Section Bariatric and Laparoscopic Surgery, University of Maryland School of Medicine, 22 S. Greene St, Room N4E27 Baltimore, Maryland, USA, E-mail: elomenzo@smail.umaryland.edu

Received February 25, 2012; Accepted February 29, 2012; Published March 05, 2012

Citation: Menzo EL (2012) Biomarkers and Obesity. J Mol Biomark Diagn 2:e103. doi:10.4172/2155-9929.1000e103

Copyright: @ 2012 Menzo EL. This is an open-access article distributed unde the terms of the Creative Commons Attribution License, which permits unrestricted use, distribution, and reproduction in any medium, provided the original author and source are credited 